Draft version 28.06.2021 submitted for peer review.

Please do not circulate or cite without the authors' permission

\title{
Confidence in complex risk judgments: the roles of uncertainty, experience, and affect
}

Matthew B. Stephensen ${ }^{1}$, Torsten Martiny-Huenger ${ }^{1}$, and Christin Schulze ${ }^{2,1}$

${ }^{1}$ UiT The Arctic University of Norway, Troms $\varnothing$, Norway

${ }^{2}$ Max Planck Institute for Human Development, Berlin, Germany

Authorship contribution statement: Author order reflects relative contribution

Open Practices Statement: Data, analysis scripts, and study materials are available at https://osf.io/ba6eh/. None of the studies were preregistered.

Corresponding author: matthew.stephensen@uit.no; matthew.stephensen@ gmail.com 


\begin{abstract}
Disagreement persists about the origin of confidence and the internal signals that influence its formation. Using combined individual participant data from four studies $(N=181)$, we examined confidence in relation to the perceived source of uncertainty for a risk judgment and explored the roles of domain-specific experience and affective evaluations in the formation of confidence. In each study, participants with domain-specific experience (backcountry skiers) performed complex risk judgments (judging avalanche risk) for multiple highly uncertain contexts (hypothetical scenarios in avalanche terrain). We examined whether more experienced participants could better recognize the inherent uncertainty of the decision environment, and if they did so with greater confidence. For complex tasks such as judging avalanche risk, experience should increase a person's understanding of the probabilistic, unpredictable nature of that environment. Yet our findings suggests that participants of all levels of experience attributed uncertainty to their own judgment process rather than to the limitations and inherent uncertainty of the environment. We also examined whether participants' affective evaluations influenced confidence in their risk judgments. Affective evaluations are understood to play a crucial orienting role in the risk judgment process. We found evidence of an interplay between affective and cognitive judgments in the formation of confidence. Participants were more confident when their affective evaluation matched their risk judgment, and less confident when there was a mismatch between the two. Our research illustrates a troubling limitation in the development of confidence with experience and the potential (dis)advantageous effect of affective evaluations on confidence in certain contexts.
\end{abstract}

Keywords: Judgment and decision making, metacognition, epistemic uncertainty, aleatory uncertainty, integral affect 


\section{Confidence in complex risk judgments: the roles of uncertainty, experience, and affect}

Doubt is unpleasant, but certainty is absurd

-Voltaire

\section{Introduction}

Judging risk involves evaluating the available evidence to infer the likelihood of a negative outcome. Faced with uncertainty, people must have confidence in the accuracy of their risk judgments in order to act (Fitzgerald et al., 2017; Gill et al., 1998; Koriat \& Goldsmith, 1996). Yet despite its importance, contention persists about the origin of confidence and the internal signals that influence its formation (Boldt et al. 2017; Meyniel et al., 2015; Petrusic \& Baranski, 2003; Yeung \& Summerfield, 2012). Although confidence has been extensively investigated using a variety of simple decision tasks, such as confidence in trivia knowledge, the applicability of this research to real-world judgements remains unclear (Dhami et al., 2004; Juslin et al., 2000; Koehler et al., 2002; Yeung \& Summerfield, 2012). The complex, goal-oriented judgment tasks that typify human behavior often involve various sources of uncertainty and a multiplicity of cognitive processes and internal signals. In the present research, we examined the formation of confidence in relation to the perceived source of uncertainty for a complex risk judgment task, and explored the roles of domain-specific experience and affective evaluations in that formation of confidence.

To capture the complexity and uncertainty of real-world decision environments, we asked backcountry skiers with varying levels of experience to judge the risk of snow avalanches in a series of uncertain hypothetical skiing scenarios modelled on the natural decision environment. Judging avalanche risk is a complex task for which no single cue or combination of cues allows perfect predictions (Landrø et al., 2020). The reliability and validity of the available evidence are often limited, ambiguous, or conflicting. Consequently, there is always some uncertainty when assessing avalanche risk (Landrø et al., 2020). One 
might reasonably expect experienced backcountry skiers to confidently recognize that in many cases, avalanche risk in a complex environment is inherently probabilistic. By examining their confidence in relation to their risk judgments, we sought to ascertain whether they indeed recognized these situations as fundamentally uncertain with regard to the decision environment, and to investigate how their perception of uncertainty relates to their experience with the decision environment.

Uncertainty can take two qualitatively distinct forms based on its source. Internal uncertainty (i.e., epistemic uncertainty) is rooted in a decision maker's incomplete knowledge, skill, or information, while environmental uncertainty (i.e., aleatory uncertainty) stems from the stochastic nature and inherent unpredictability of the environment (Fox \& Ülkümen, 2011; Gillies, 2000; Kahneman \& Tversky, 1982; Kozyreva \& Hertwig, 2021; Løhre \& Teigen, 2016; Peterson \& Pitz, 1988; Teigen \& Løhre, 2017; Ülkümen et al., 2016). Each form of uncertainty elicits a different type of probabilistic judgment. Internal uncertainty evokes an epistemic probabilistic judgment that involves reporting a subjective degree of belief in a predicted outcome, whereas environmental uncertainty evokes an aleatory probabilistic judgment that involves estimating the long-run frequency of an outcome given the external conditions (Fox \& Ülkümen, 2011; Kozyreva \& Hertwig, 2021; Løhre \& Teigen, 2016; Ülkümen et al., 2016). The perception of uncertainty and corresponding interpretation of the judgment task is highly subjective and contextual. People can disagree about the source of uncertainty for a particular judgment task, and a given individual can perceive different sources of uncertainty between specific instances of a category of judgment task (Fox \& Ülkümen, 2011, Ülkümen et al., 2016).

Crucially, confidence in a risk judgment provides insight as to how a decision maker perceives the uncertainty of that judgment task. Because people make different probabilistic judgments in response to either form of uncertainty, the meaning of what is reported on a risk 
rating scale differs according to how the uncertainty of the judgment task is perceived (Fox \& Ülkümen, 2011; Løhre \& Teigen, 2016; Teigen \& Løhre, 2017; Ülkümen et al., 2016). That meaning can be inferred based on the retrospective confidence judgment. If a person attributes the uncertainty of judging avalanche risk to their lack of knowledge and/or inadequacy of their judgment strategy — interpreting the task as an epistemic probabilistic judgment — then the reported risk value reflects their subjective degree of belief in the safety of the scenario (Fox \& Ülkümen, 2011; Løhre \& Teigen, 2016). Since confidence is equivalent to degree of belief for epistemic judgments, we would expect confidence to track reported risk values: confidence should be highest at either limit of the response scale where the degree of belief in the safety of the scenario is highest, and lowest at the midpoint of the response scale where the degree of belief is lowest. By contrast, if a person attributes uncertainty to the stochastic nature of the decision environment—interpreting the task as an aleatory probabilistic judgment - then reported risk reflects the long-run frequency of a safe outcome given the evidence (Fox \& Ülkümen, 2011; Løhre \& Teigen, 2016). One can be more or less confident in any aleatory probabilistic estimate of the state of the world if the evidence supports that judgement. For example, when randomly drawing a card from a full deck, one can be highly confident that $25 \%$ of the time the suit of the card will be hearts. We could therefore expect equal confidence ratings across all reported risk values. The two forms of uncertainty evoke different probabilistic judgments and, correspondingly, different confidence judgments. By examining retrospective confidence reports in relation to what is reported on the risk rating scale, we can infer how participants perceived the uncertainty of the risk judgment. This is important because for complex tasks such as judging avalanche risk, people should ideally understand that the decision environment is probabilistic and that correctly judging the outcome is not exclusively dependent upon a person's knowledge and judgment strategy. 
Domain-specific experience should play an important role in a person' ability to more confidently recognize the probabilistic, unpredictable nature of the decision environment. However, although experience is often intuitively associated with expertise, the relationship between experience, confidence, and the degree to which a judgment corresponds with reality is not straightforward. As a person gains domain-specific experience, their judgment strategy tends to become more established, resulting in greater confidence (Fischer \& Budescu, 2005; Sanchez \& Dunning, 2018, 2020). We might therefore expect experienced backcountry skiers to have an increased understanding of the inherent environmental uncertainty when judging avalanche risk. Yet greater experience and confidence do not guarantee that that judgment strategy represents a correct model of the world (Einhorn \& Hogarth, 1978; Koriat, 2008; Hogarth et al, 2015). The acquisition of experience increases the conviction and tenacity with which a person holds to a judgment strategy more rapidly than it refines the content of that judgment strategy (DePaulo \& Pfeifer, 1986; Sanchez \& Dunning, 2018, 2020). We therefore examined whether more experienced individuals were better able to recognize the inherent environmental uncertainty when judging avalanche risk, and if they had greater confidence in that recognition.

Beyond this examination of confidence in relation to the perceived source of uncertainty, we also explored whether a backcountry skier's affective evaluation of a scenario influenced their confidence in their avalanche risk judgment. Affective evaluations serve as a valuable and compelling orienting mechanism in the perception of risk (Blanchette \& Richards, 2010; Finucane et al, 2000; Lerner et al., 2015; Lowenstein et al., 2001; Schwarz, 2012; Slovic, 1987; Slovic et al., 2002, 2004). Given that relation, it is reasonable to assume that affective evaluations might also influence the formation of confidence in risk judgments. That mood (i.e., incidental affect) was found to influence confidence (Koellinger \& Treffers, 2015; Kuvaas \& Kaufmann, 2004; Massoni, 2014; Prinz et al., 2019; Sidi et al., 2018) is 
further reason to suspect that affective evaluations (i.e., integral affect) are an important internal signal during the formation of confidence. We conceptualize affective evaluations as a generalized positive or negative response to the target of judgment, which we measured as scenario liking. Liking has a motivational component that is relevant for the risk judgment process: people are more likely to approach liked and avoid disliked stimuli (Chen \& Bargh, 1999). Generally, liking positively relates to perceived safety. The more an individual likes (i.e., approach motivation) a scenario, the safer it is perceived, whereas the more they dislike (i.e., avoid motivation) a scenario, the more dangerous it is perceived (Alhakami \& Slovic, 1994; Finucane et al., 2000; Slovic et al., 2002; Stephensen \& Martiny-Huenger, 2021). Yet despite this general relation, it is possible that an individual likes a scenario that is perceived as dangerous or dislikes a scenario perceived as safe. We explored whether congruence (e.g., liking a scenario perceived as safe) and incongruence (e.g., liking a scenario perceived as dangerous) between affective evaluations and risk judgments influenced the formation of confidence.

\section{Methods}

Participants. We obtained data from one-hundred and eighty-one individuals (59 women, 122 men, $M_{\text {age }}=31$ years, $S D=11.18$ years, range $=15-63$ years) who participated in one of four independent studies. As explained below when reporting our methods of analysis, we combined the individual participant data from those four studies for pooled analysis. Participants in Studies $1(N=40)$ and $3(N=54)$ were backcountry skiers from Norway who did not receive compensation for participating. Participants in Study $4(N=41)$ were backcountry skiers from the western USA who received compensation for participation. To obtain data from participants with a wide range of experience, participants in Study $2(N=$ 46) were a mixed sample comprising backcountry skiers from Norway (37\% of participants) who did not receive compensation for participating, and psychology students at a Norwegian 
university (63\% of participants) who received course credit for participating. The backcountry skier participants in each study had experience in performing similar risk judgments in preparation for and during actual ski tours in avalanche terrain. Arguably, the activity of backcountry skiing, the environment of avalanche terrain depicted in the scenarios, and the task of judging avalanche risk were familiar, meaningful, and important for those experienced participants.

We obtained informed consent from all participants in each study. All studies were conducted in accordance with the ethical research protocols of UiT - The Arctic University of Norway and the Norwegian Center for Research Data (NSD ref. numbers 54651 and 58839). Data on judged safety, scenario liking, and participant experience from Studies 2 to 4 were previously published in the context of testing a hypothesized relation between scenario liking and judged safety (see Stephensen \& Martiny-Huenger, 2021). However, confidence did not pertain to the focus of that investigation; we neither analyzed nor published data on confidence from any study in that article.

Materials. We conducted all four studies online using the jspsych programme (de Leeuw, 2015). We developed hypothetical scenarios each depicting a distinct backcountry ski tour on a snow-covered mountain slope (i.e., avalanche terrain, see Figure 1 for an example). Each scenario began with a description of the tour that included basic information on the terrain, elevation, average and maximum slope steepness, and the current weather. A photograph of the mountain slope was located below the tour information. Beneath the photograph was the forecasted regional avalanche danger level (5-category scale), the current avalanche problem(s) caused by the combination of weather and snow conditions, and the weather history. The avalanche danger scale and the avalanche problems are standardized tools used by the Norwegian Avalanche Warning Service (Varsom), the European Avalanche Warning Services (EAWS), the American Avalanche Association, and the National 
Avalanche Center in the USA. The photograph and textual content of each scenario provided objective information for judging the degree of avalanche risk (i.e., judged safety).

All the hypothetical scenarios represent realistic situations typically encountered in avalanche terrain. We designed each of the scenarios to have low predictability and high environmental uncertainty. None of the scenarios included indicators of either very low or very high risk of avalanche. The available information cues in the photo and textual content were ambiguous and/or conflicting. For example, although the danger level of 3-considerable (on a five-category scale), the weather history, and the prevailing avalanche problems in the scenario depicted in Figure 1 together indicate an increased probability of an avalanche, the terrain features, low slope angle, and the lack of any signs in the photograph indicative of the presence of the avalanche problems indicate a reduced probability of avalanche. It was not possible to ascertain that any scenario was safe or dangerous. Each scenario was marked by high environmental uncertainty.

Studies 1, 2, and 3 used the same six scenarios. For Study 4, we used six new scenarios. The content of all the scenarios such as the measurement units (e.g., Celsius and meters vs Fahrenheit and feet), geography, tour descriptions, and photographs were suited to the norms and familiar conditions of the participants. We based the content and format of the avalanche hazards information on historic avalanche forecasts from the Norwegian Avalanche Warning Service (Varsom, n.d.) and from local US avalanche warning services (Colorado Avalanche Information Center, n.d.; Gallatin National Forest Avalanche Center, n.d.; Utah Avalanche Center, n.d.). All scenarios used in the four studies are available on the Open Science Framework at https://osf.io/ba6eh/. ${ }^{1}$

\footnotetext{
${ }^{1}$ We prepared two versions of each of the scenarios: one version included the clearly visible ski tracks on the mountain slope in the scenario photograph while the other version did not include ski tracks in the photograph (see Figure 1 for an example). As reported in Stephensen and Martiny-Huenger (2021), this within-subject manipulation (the presence vs. absence of ski tracks) was an attempt to manipulate scenario liking while holding constant the objective risk level of each scenario. However, this
} 


\section{Figure 1}

Example of the backcountry skiing scenarios judged by participants.

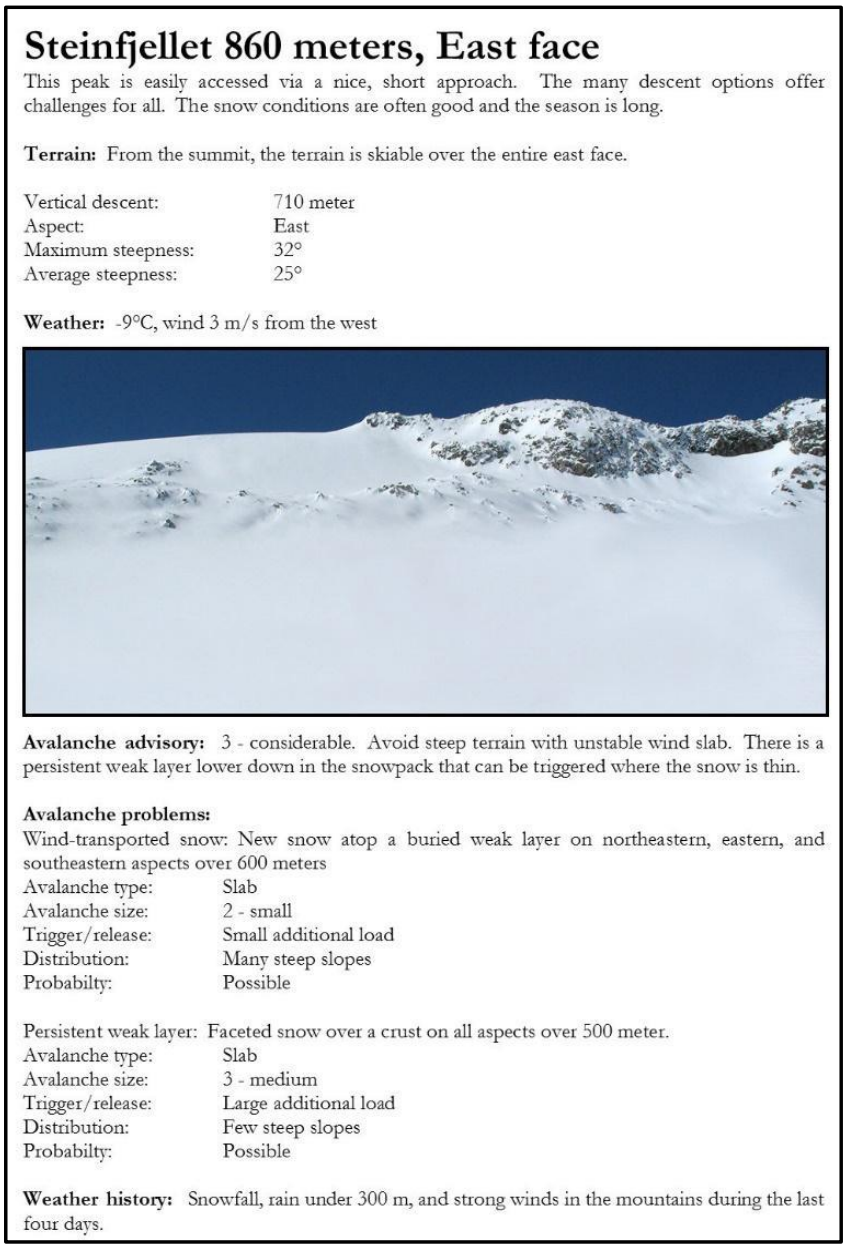

Procedure. Participants accessed the online study via a web browser at a time and device of their choosing. Each scenario was presented on a unique webpage. The questions were presented immediately below each scenario on that same webpage. All the questions and response scales for each scenario were presented simultaneously and could be answered in any order. Participants in all four studies judged the safety of each scenario in terms of the avalanche risk, referred to as judged safety, and reported their retrospective confidence in

manipulation failed to influence risk judgments, confidence, or affective evaluations. We therefore do not investigate or discuss this manipulation further in this article. 
judged safety, referred to as confidence. Participants in studies 2 to 4 also reported their affective evaluation of each scenario, referred to as scenario liking. For judged safety, participants answered three questions following the general statement "Regarding the avalanche risk...": 1) "Is it safe to ski the slope in these conditions?" 2) "Is it dangerous to ski the slope in these conditions?" (reverse-coded), and 3) "Is the snowpack stable enough to ski this slope?" On a 7-point scale labeled "Not at all" and "Very much" at the extreme points. For the degree of liking the scenario, following the general statement "Regarding your desire to ski this slope, the slope appears..." participants in Studies 2, 3, and 4 rated the scenario according to the following three adjectives: 1) "Attractive", 2) "Uninteresting" (reverse-coded), and 3) "Enjoyable" on a 7-point scale labeled "Not at all" and "Very much" at the extreme points. We did not assess scenario liking in Study 1. For the degree of confidence in judged safety, participants answered three questions following the general statement "Regarding your judgments of the avalanche risk...": 1) "Are you confident in your assessment of the avalanche risk?"2) "Do you doubt your judgment of the avalanche danger?" (reverse-coded), and 3) "Are you confident in your judgment of the safety of the snowpack?" On a 7-point scale labeled "Not at all" and "Very much" at the extreme points. Participants were required to answer all questions for each scenario to proceed with the study. It was not possible to return to earlier scenarios. Participants in Study 1 judged each of the six available scenarios. Participants in Studies 2, 3, and 4 judged four scenarios from the sample of scenarios for their respective region. Selections were random for each participant. Upon completing the scenarios, participants reported their age and gender. Participants then reported their skiing ability, avalanche training, years of backcountry skiing experience, and average number of backcountry skiing days per season, each of which was measured on a 7point scale. 
Analysis. To synthesize the evidence across all studies and more accurately estimate parameters for any effect, we combined the individual participant data from each of the four studies for pooled analysis (da Costa \& Sutton, 2019). The dataset comprises 804 scenario evaluations from the four studies. We calculated mean scores for confidence (three items, Cronbach's alpha $=.89, N=804, M=4.62, S D=1.42$ ), judged safety (three items, Cronbach's alpha $=.92, N=804, M=3.18, S D=1.44$ ), and scenario liking (three items, Cronbach's alpha $=.90, N=564, M=5.11, S D=1.53$ ) per participant and scenario. We calculated a mean experience score per participant from the measurements of skiing ability, avalanche training, years of backcountry skiing experience, and average number of backcountry skiing days per season (four items, Cronbach's alpha $=.83, N=181, M=4.46$, $S D=1.55)$. Three participants did not provide responses to all four measures of experience so we calculated mean experience scores using the available measures for those participants. We standardized the confidence, judged safety, scenario liking, and participant experience scores for all analyses.

We first analyzed whether an increase of experience corresponds to a similar increased ability to distinguish the source of uncertainty for a judgment task. We tested the variance of judged safety scores across all levels of participant experience for each scenario to determine if judged safety scores clustered among more experienced participants. We used linear regression analysis to determine whether experience predicts judged safety for each scenario. We used Breusch-Pagan tests of non-constant variance for that linear relation to determine if variance in judged safety was constant across participant experience levels for each scenario.

To investigate a possible U-shaped relation between judged safety and confidence, we used two-line regression analysis (Simonsohn, 2018) for all models predicting confidence. A U-shaped relation involves a sign change in the regression coefficient between confidence and the predictor variable. The point at which the regression coefficient changes between positive 
and negative is referred to as the breakpoint. We set the breakpoint at the midpoint of the judged safety reporting scale (safety judgment $=4$ on a scale from 1 to 7 ). We split the data into two ranges according to the unstandardized judged safety score: one range in the direction of dangerous for judged safety scores $<4$ and a second range in the direction of safe for judged safety scores $\geq 4$. We fit identical models - as described in the next paragraph - for each of these ranges of judged safety.

We examined how confidence relates to the distribution of judged safety and any influence that experience and liking have on that relation. We estimated the effect of judged safety on confidence moderated by participant experience (confidence $\sim$ judged safety * participant experience $+(1 \mid$ participant $)+(1 \mid$ scenario $)+(1 \mid$ study $))$ using the pooled data from all four studies. We then investigated the role of scenario liking in the formation of confidence using the combined data from Studies 2, 3, and 4. We excluded data from Study 1 in that analysis because it does not include the liking measure. We estimated the effect of scenario liking on confidence when controlling for the effects of judged safety and experience $($ confidence $\sim$ judged safety $*$ participant experience + scenario liking $+(1 \mid$ participant $)+$ $(1 \mid$ scenario $)+(1 \mid$ study $))$

To estimate the parameters of those models, we fit Bayesian linear mixed models to evaluate the probability of the predictor variable(s) to predict the response variable confidence given the data. An advantage of Bayesian methods is the incorporation of (un)certainty caused by the amount of data, which is reflected in the width of the posterior distribution(s) of the model parameters (Nalborczyk et al., 2020). We used the brms package (Bürkner, 2017, 2018) in R (R Core Team, 2017) to generate posterior probability distributions to calculate median-based regression coefficients and 95\% highest density intervals (HDI) to estimate parameters for each effect. We included varying intercepts for participants and scenarios in the models to account for by-subject and by-scenario variability. We also included varying 
intercepts for studies in the models to account for by-study clustering and the potential heterogeneity of effect parameter values between studies in the combined data. We defined a weakly informative prior distribution (Gelman et al., 2008, 2017) for the predictor judged safety (Normal $(.2,1)$ for judged safety $\geq 4$, Normal(-.2,1) for judged safety $<4$ ) based on the assumption of a correlation between confidence and the extremeness of judged safety. We defined uninformed prior distributions $(\operatorname{Normal}(0,10))$ for the possible effects of scenario liking and participant experience. We checked that Rhat values were less than 1.05 and greater than .9 to ensure chain convergence for all parameters of interest. Diagnostics indicated that all Rhat values were either 1.000 or 1.001 for all examined effects. The data and $\mathrm{R}$ script for data processing and analysis are publicly available on the Open Science Framework (https://osf.io/ba6eh/).

\section{Results}

Participants disagreed about the avalanche risk of each scenario. Figure 2 illustrates that judged safety scores per scenario were uniformly distributed across the response scale. Participants judged the risk heterogeneously, ranging from clearly safe to clearly dangerous, rather than clustering at the midpoint of the response scale. How then did confidence relate to that distribution of judged safety scores? As Figures 3 and 4 illustrate, confidence was lowest at the midpoint of the response scale and increased as judged safety became more extreme, be it in the direction of safe or dangerous. Table 1 presents the posterior distributions of all parameter estimates for the relation between judged safety and confidence. Participants reported unequal confidence across all values of judged safety. There was a U-shaped relation between judged safety and confidence.

Domain-specific experience did not reduce the variability of judged safety of each scenario. Linear regression and Breusch-Pagan tests (see the supplemental materials for results) indicated that experience did not predict judged safety and that the variance in judged 
safety remained constant across participant experience levels for all scenarios (except scenario 1 used in Studies 1 to 3). Although judged safety scores did not converge around the midpoint of the response scale at greater experience levels, experience magnified confidence in those judgments. Table 1 presents the posterior distributions of all parameter estimates for the influence of experience on confidence. As Figure 3 illustrates, higher participant experience predicted higher confidence, irrespective of judged safety values. Yet experience did not moderate the U-shaped relation between judged safety and confidence.

Scenario liking predicted confidence. Figure 4 illustrates that the effect of scenario liking on confidence was specific to whether a scenario was judged in the direction of safe or dangerous. Table 1 presents the posterior distributions of all parameter estimates for the relations between scenario liking and confidence. When a scenario was perceived as dangerous to some degree (judged safety < 4), confidence decreased with higher scenario liking. However, when a scenario was perceived as safe to some degree (judged safety $\geq 4$ ), confidence increased with higher scenario liking. Liking a scenario perceived as safe or disliking a scenario perceived as dangerous increased confidence. By contrast, liking a scenario perceived as dangerous or disliking a scenario perceived as safe reduced confidence in the risk judgment. 


\section{Figure 2}

Distribution of judged safety scores per scenario. Scenarios 1 to 6, which were used in Studies 1 to 3 (640 observations), show highly uniform distributions of judged safety scores. The distribution of judged safety scores for scenarios 7 to 12, which were only used in Study 4 (164 observations), are less uniform but nonetheless exhibit a large range of values indicating disparity in the perceived safety of each scenario.

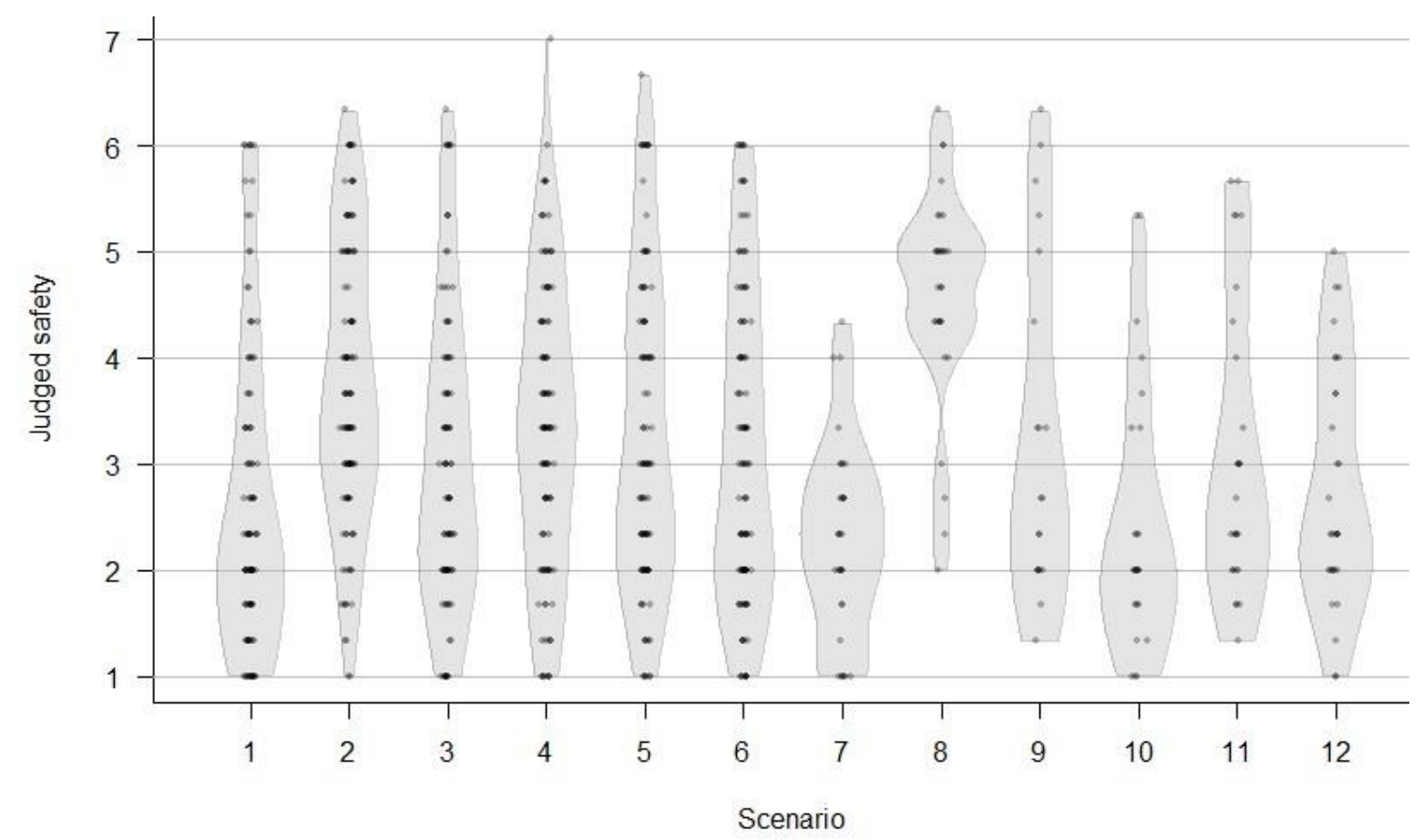




\section{Figure 3}

The effects of judged safety and participant experience on confidence (confidence $\sim$ judged safety * participant experience) when a scenario is perceived as dangerous to some degree (plot on left, judged safety <4) or perceived as safe to some degree (plot on right, judged safety $\geq 4)$. The effect of judged safety is plotted on the $x$-axis. The effect of participant experience is plotted as a dotted line for -1 SD participant experience, a solid line for mean participant experience, and a dashed line for +1 SD participant experience.
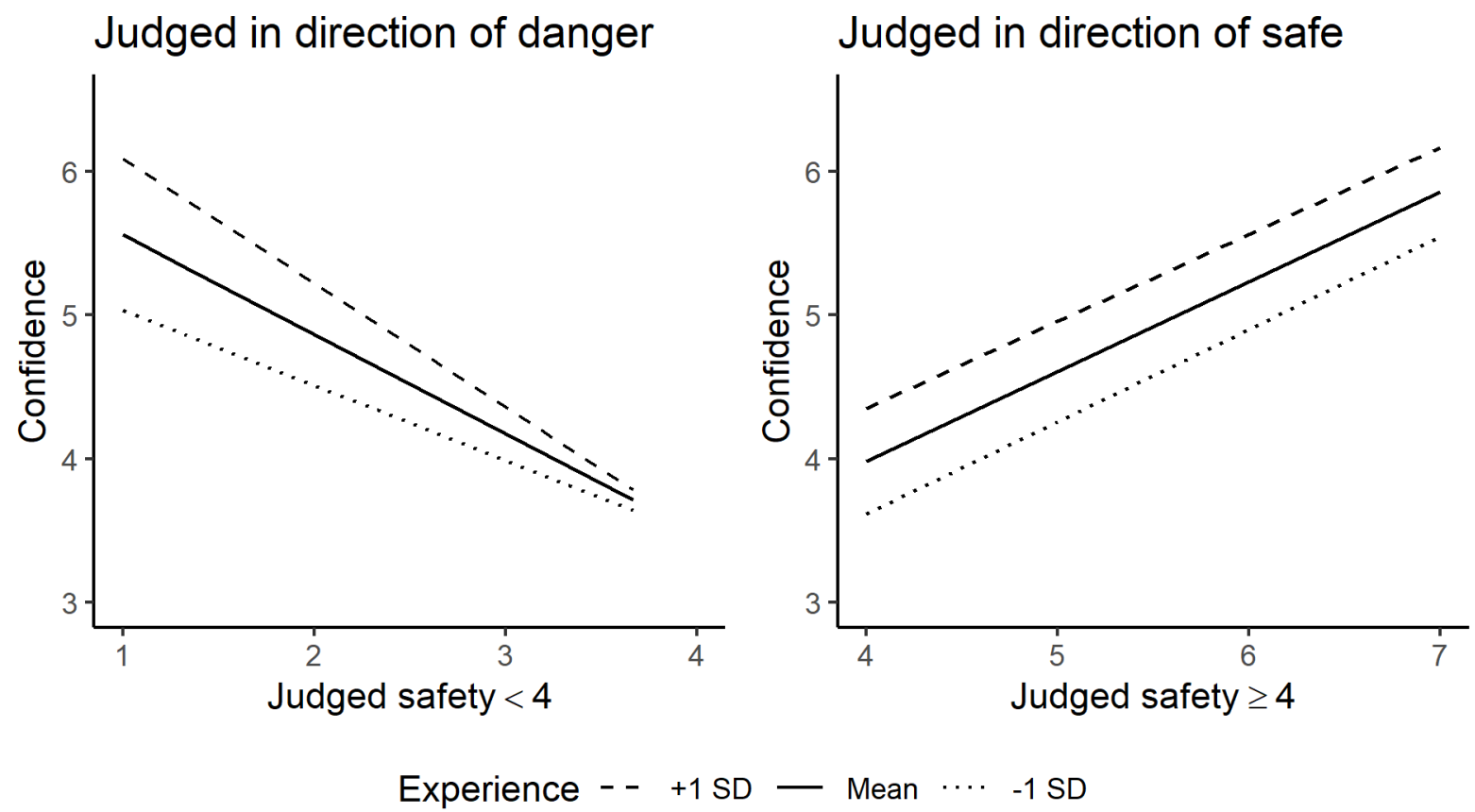


\section{Figure 4}

The effects of judged safety, scenario liking, and participant experience on confidence (confidence $\sim$ judged safety $*$ participant experience + scenario liking) when a scenario is perceived as dangerous to some degree (plot on left, judged safety $<4$ ) or perceived as safe to some degree (plot on right, judged safety $\geq 4$ ). The effect of judged safety is plotted on the $x-$ axis. The effect of participant experience is plotted as a dotted line for - 1 SD participant experience, a solid line for mean participant experience, and a dashed line for $+1 \mathrm{SD}$ participant experience. The effect of scenario liking is faceted into -1 SD scenario liking in the left column of each plot, mean scenario liking in the middle column of each plot, and +1 SD scenario liking in the right column of each plot.
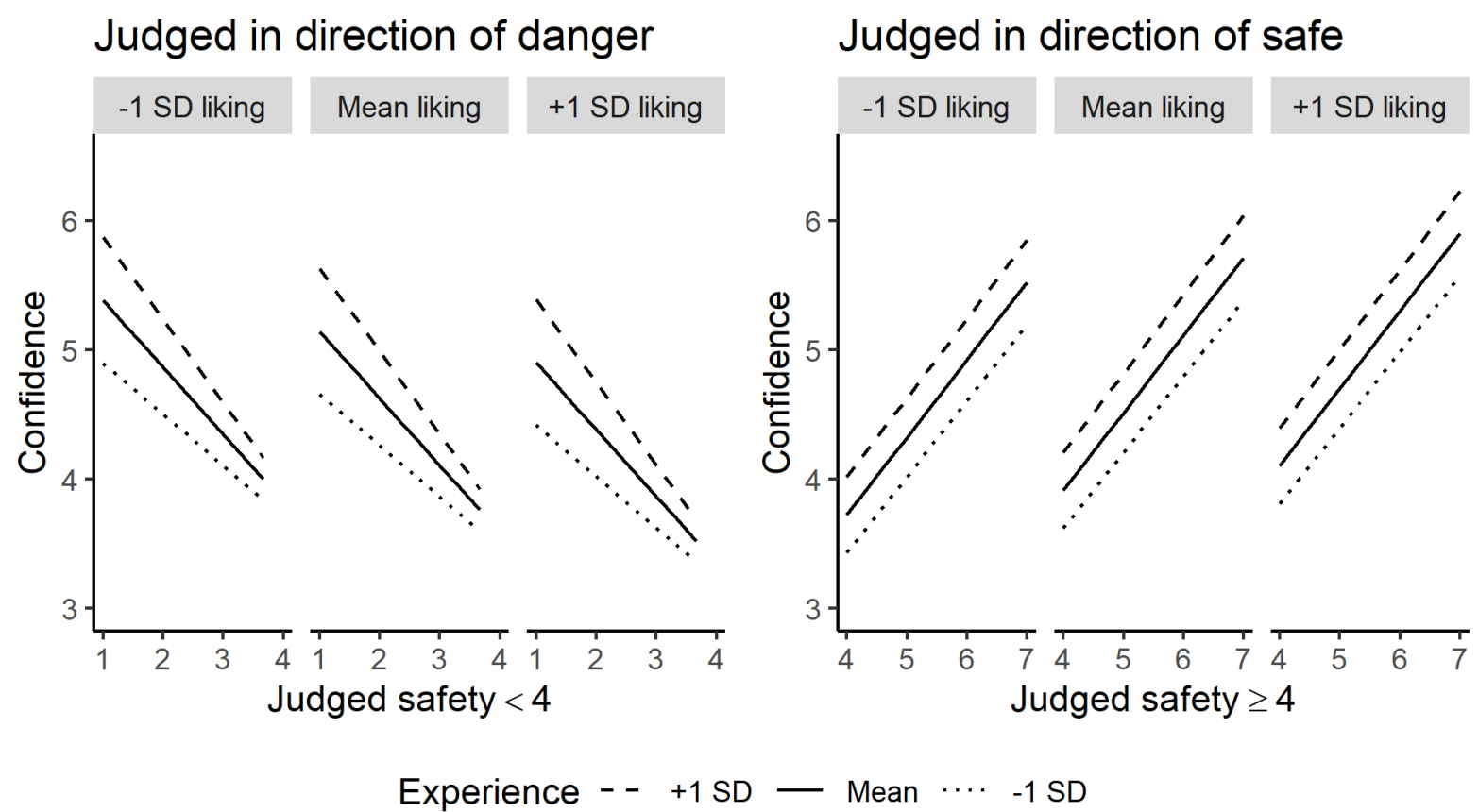


\section{Table 1}

Posterior parameter distributions of the effects predicting confidence in the judgment that a scenario is dangerous to some degree (judged safety $<4$ ) or is safe to some degree (judged safety $\geq 4)$.

\begin{tabular}{lrrrr}
\hline \multirow{2}{*}{ Effect } & \multicolumn{2}{c}{ Judged safety < 4} & \multicolumn{2}{c}{ Judged safety $\geq 4$} \\
\cline { 2 - 5 } Confidence $\sim$ judged safety * experience & \multicolumn{1}{c}{$b(S E)$} & $95 \%$ HDI & $b(S E)$ & $95 \%$ HDI \\
$\quad$ Judged safety & $-.69(.06)$ & $-.82,-.57$ & $.63(.08)$ & $.47, .79$ \\
Experience & $.10(.08)$ & $-.05, .25$ & $.28(.11)$ & $.06, .50$ \\
Judged safety * experience & $-.17(.06)$ & $-.28,-.06$ & $-.02(.08)$ & $-.18, .14$ \\
Confidence $\sim$ judged safety * experience & + scenario liking & & \\
Judged safety & $-.55(.08)$ & $-.71,-.39$ & $.61(.10)$ & $.42, .80$ \\
Experience & $.15(.09)$ & $-.02, .33$ & $.22(.13)$ & $-.04, .47$ \\
Scenario liking & $-.16(.05)$ & $-.26,-.05$ & $.19(.08)$ & $.04, .34$ \\
Judged safety * experience & $-.12(.07)$ & $-.25, .02$ & $.01(.09)$ & $-.17, .19$ \\
\hline
\end{tabular}

\section{Discussion}

Experience with a judgment task ideally should reduce internal, epistemic uncertainty by increasing a person's task-relevant knowledge and the accuracy of their judgment strategy. In a highly complex, uncertain decision environment, experience should also increase a person's understanding of the probabilistic nature of that environment. More experienced backcountry skiers should be better able to recognize when the complexity of the environment exceeds their ability to reliably determine whether the conditions are safe or dangerous. However, that is not what we found. Confidence in avalanche risk judgments increased with domain-specific experience, echoing previous findings on the development of confidence (Fischer \& Budescu, 2005; Sanchez \& Dunning, 2018, 2020). Yet that greater confidence did not correspond to an increased ability to recognize the inherent uncertainty of the scenarios. More experienced participants were not better able to understand that the safety or danger of each scenario could not be established. Our pattern of findings suggests that participants of all 
levels of experience attributed uncertainty to their own judgment process rather than to the limitations and inherent uncertainty of the decision environment.

That people fail to recognize the unpredictability of the decision environment and instead attribute uncertainty to their own incomplete knowledge, competency, or information is problematic from a policy perspective. Ideally, backcountry skiers in avalanche terrainand decision makers in other contexts performing similarly complex risk judgments—-should understand when the safety or danger of a situation cannot be established because of the inherent uncertainty of the decision environment. They should not mistakenly assume that safety or danger can always be determined or that they have the expertise to outthink the information limits and uncertainty of the environment. Unfortunately, the experience of avalanche terrain appears to lead many backcountry skiers to make such mistaken assumptions. Avalanche terrain is a decision environment in which poor decisions are infrequently marked by valid feedback (Ebert, 2019; Johnson et al., 2020; Zweifel \& Haegeli, 2014). Valid feedback is essential for the experiential learning and calibration process (Fischer \& Budescu, 2005; Hogarth et al., 2015; Sanchez \& Dunning, 2018, 2020). The absence of valid performance feedback makes it problematic for decision makers to evaluate their performance and refine their judgment strategy. This obstructs skill development and magnifies the likelihood of overconfidence in erroneous decision making practices (Hogarth et al., 2015). Although confidence increased with domain-specific experience, even the most experienced participants did not confidently recognize that the conflicting, ambiguous evidence made it impossible to establish the safety or danger of any scenario. In this regard, experience did not correspond to expertise.

We recognize that the design of the scenarios and the studies might offer alternative explanations for the high variance in judged safety across all levels of experience, and for the perceived source of uncertainty. The use of exclusively uncertain scenarios may have been 
ecologically invalid for an experienced participant sample (Dhami et al, 2004). In the real world, experienced participants will have previously encountered situations that are objectively safe or dangerous among other situations that are uncertain. The distribution of reported judged safety and the confidence in those judgments per participant may partly reflect the probabilistic distribution (i.e., base rate) of safety, danger, and uncertainty encountered in their own real-world experience. However, had ecological validity been crucial, we would have expected less experienced participants to have shown less variance in their risk judgments because their prior assumptions about base rates of safe, uncertain, and dangerous conditions are likely weaker. That they did not suggests that the lack of ecological validity was not the main cause for our results.

Another possible limitation concerns the interpretation of confidence to infer the perceived source of uncertainty. By asking participants about their confidence, we may have prompted them to assess their degree of belief in their risk judgment rather than the likelihood of their risk judgment being correct. Previous research (Fox \& Ülkümen, 2011; Løhre \& Teigen, 2016; Ülkümen et al., 2016) has shown that people's choice of language when communicating uncertainty differs as a function of where they locate the source of uncertainty. Aleatory or environmental uncertainty is expressed in likelihood statements (e.g., "I'd say there is a $90 \%$ chance," or "I think there is a high probability") whereas epistemic uncertainty is expressed in confidence statements (e.g., "I am $80 \%$ sure," or "I'm reasonably certain"). Because the perception of uncertainty influences the choice of language, one might conversely assume that the language used to formulate a judgment task could influence the perception of uncertainty. By using the language of confidence when asking participants to retrospectively judge the accuracy of their risk judgments, we may have made epistemic uncertainty more salient. However, the relation between language and perceived uncertainty was found when the choice of language pertained to judging a possible outcome or state (e.g., 
"I am _ \% sure that Germany will win the FIFA World Cup" versus "I think there is a _ $\%$ chance that Germany will win the FIFA World Cup"). That relation has not been demonstrated (or, to the best of our knowledge, investigated) when the choice of language pertains to retrospective judgments of performance (i.e., metacognitive judgments), which are different from judging a possible outcome.

The final question we examined concerned whether affective evaluations influenced confidence. Our research found evidence of an interplay between affective and cognitive judgments in the formation of confidence. Judged safety and scenario liking each served as a cue for the formation of confidence, and their relative configuration introduced additional variability into confidence reports. Agreement between the cognitive and affective evaluations (liking scenarios perceived as safe, disliking scenarios perceived as dangerous) magnified confidence whereas disagreement between the two (liking scenarios perceived as dangerous, disliking scenarios perceived as safe) decreased confidence. This (in)congruity effect is likely beneficial under uncertainty. Confidence functions as a control process for whether a decision is translated into action (Fitzgerald et al., 2017; Gill et al., 1998; Koriat \& Goldsmith, 1996). Cognitive and affective evaluations appear to check and balance each other to prevent overconfidence and mistaken actions. A disagreement between cognitive and affective evaluations would suggest that either evaluation overlooked or misjudged a valid, relevant cue(s) or mistakenly relied on an invalid, irrelevant cue(s). If both cognitive and affective evaluation processes are well calibrated for a judgment task, reduced confidence when there is disagreement between the two would be highly advantageous because it would decrease the likelihood of acting on mistaken evaluations. However, if an affective evaluation is based on irrelevant contextual features, a positive affective response could diminish confidence in an objectively correct judgment of danger. For example, if a backcountry skier correctly judges that a ski tour is unsafe but nonetheless likes it because of its aesthetic beauty or popularity, 
that incongruity would undermine confidence in their accurate risk judgment and, perhaps, their decision to not ski.

In conclusion, our research provides insight into several internal signals that influence the formation of confidence in complex risk judgments. Specifically, our findings illustrate a troubling limitation of the development of confidence with experience and the potential (dis)advantageous effect of affective evaluations on confidence in certain contexts. 


\section{References}

Alhakami, A. S., \& Slovic, P. (1994). A psychological study of the inverse relationship between perceived risk and perceived benefit. Risk Analysis, 14(6), 1085-1096. https://doi.org/10.1111/j.1539-6924.1994.tb00080.x

Blanchette, I., \& Richards, A. (2010). The influence of affect on higher level cognition: A review of research on interpretation, judgment, decision making and reasoning. Cognition and Emotion, 24(4), 561-595. https://doi.org/10.18637/jss.v067.i01

Boldt, A., de Gardelle, V., \& Yeung, N. (2017). The impact of evidence reliability on sensitivity and bias in decision confidence. Journal of Experimental Psychology: Human Perception and Performance, 43(8), 1520-1531. https://doi.org/10.1037/xhp0000404

Bürkner, P-C. (2017). brms: An R package for Bayesian multilevel models using stan. Journal of Statistical Software, 80, 1-28. https://doi.org/10.18637/jss.v080.i01

Chen, M., \& Bargh, J. A. (1999). Consequences of automatic evaluation: Immediate behavioral predispositions to approach or avoid the stimulus. Personality and Social Psychology Bulletin, 25, 215-224. https://doi.org/10.1177/0146167299025002007

Colorado Avalanche Information Center. (n.d.). Forecasts. Retrieved from https://avalanche.state.co.us/

da Costa, B. R., \& Sutton, A. J. (2019). A comparison of the statistical performance of different meta-analysis models for the synthesis of subgroup effects from randomized clinical trials. BMC medical research methodology, 19(1), 198-198. https://doi.org/10.1186/s12874-019-0831-8

Dhami, M. K., Hertwig, R., \& Hoffrage, U. (2004). The role of representative design in an ecological approach to cognition. Psychological Bulletin, 130(6), 959-988. https://doi.org/10.1037/0033-2909.130.6.959 
de Leeuw, J. R. (2015). jsPsych: A JavaScript library for creating behavioral experiments in a web browser. Behavior Research Methods, 47, 1-12. https://doi.org/10.3758/s13428-014$0458-y$

DePaulo, B. M., \& Pfeifer, R. L. (1986). On-the-job experience and skill at detecting deception. Journal of Applied Social Psychology, 16(3), 249-267. https://doi.org/10.1111/j.1559-1816.1986.tb01138.x

Ebert, P. A. (2019). Bayesian reasoning in avalanche terrain: a theoretical investigation. Journal of Adventure Education and Outdoor Learning, 19(1), 84-95. https://doi.org/10.1080/14729679.2018.1508356

Einhorn, H. J., \& Hogarth, R. M. (1978). Confidence in judgment: Persistence of the illusion of validity. Psychological Review, 85, 395-416. https://doi.org/10.1037/0033295X.85.5.395

Finucane, M. L., Alhakami, A., Slovic, P., \& Johnson, S. M. (2000). The affect heuristic in judgments of risks and benefits. Journal of Behavioral Decision Making, 13(1), 1-17. https://doi.org/10.1002/(SICI)1099-0771(200001/03)13:1\%3C1::AIDBDM333\%3E3.0.CO;2-S

Fischer, I., \& Budescu, D. V. (2005). When do those who know more also know more about how much they know? The development of confidence and performance in categorical decision tasks. Organizational Behavior and Human Decision Processes, 98(1), 39-53. https://doi.org/10.1016/j.obhdp.2005.04.003

Fitzgerald, L. M., Arvaneh, M., \& Dockree, P. M. (2017). Domain-specific and domaingeneral processes underlying metacognitive judgments. Consciousness and Cognition, 49(Supplement C), 264-277. https://doi.org/10.1016/j.concog.2017.01.011

Fox, C. R., \& Ülkümen, G. (2011). Distinguishing two dimensions of uncertainty. In W. Brun, G. Keren, G. Kirkebøen, \& H. Montgomery (Eds.), Perspectives on Thinking, 
Judging, and Decision Making: A Tribute to Karl Halvor Teigen. Oslo:

Universitetsforlaget.

Gallatin National Forest Avalanche Center. (n.d.). Avalanche Forecast. Retrieved from https://www.mtavalanche.com/forecast

Gelman, A., Jakulin, A., Pittau, M. G., \& Su, Y.-S. (2008). A weakly informative default prior distribution for logistic and other regression models. The Annals of Applied Statistics, 2(4), 1360-1383, 1324. https://doi.org/10.1214/08-AOAS191

Gelman, A., Simpson, D., \& Betancourt, M. (2017). The prior can often only be understood in the context of the likelihood. Entropy, 19(10), 555. https://doi.org/10.3390/e19100555

Gill, M. J., Swann Jr, W. B., \& Silvera, D. H. (1998). On the genesis of confidence. Journal of Personality and Social Psychology, 75(5), 1101-1114. https://doi.org/10.1037/00223514.75.5.1101

Gillies, D. (2000). Philosophical theories of probability. London: Routledge.

Hogarth, R. M., Lejarraga, T., \& Soyer, E. (2015). The two settings of kind and wicked learning environments. Current Directions in Psychological Science, 24(5), 379-385. https://doi.org/10.1177/0963721415591878

Johnson, J., Mannberg, A., Hendrikx, J., Hetland, A., \& Stephensen, M. (2020). Rethinking the heuristic traps paradigm in avalanche education: past, present and future. Cogent Social Sciences, 6(1). https://doi.org/10.1080/23311886.2020.1807111

Juslin, P., Winman, A., \& Olsson, H. (2000). Naive empiricism and dogmatism in confidence research: A critical examination of the hard-easy effect. Psychological Review, 107(2), 384-396. https://doi.org/10.1037/0033-295X.107.2.384

Kahneman, D., \& Tversky, A. (1982). Variants of uncertainty. Cognition, 11(2), 143-157. https://doi.org/10.1016/0010-0277(82)90023-3 
Koehler, D. J., Brenner, L., \& Griffin, D. (2002). The calibration of expert judgment: heuristics and biases beyond the laboratory. In D. Griffin, D. Kahneman, \& T. Gilovich (Eds.), Heuristics and Biases: The Psychology of Intuitive Judgment (pp. 686-715). Cambridge: Cambridge University Press.

Koellinger, P., \& Treffers, T. (2015). Joy leads to overconfidence, and a simple countermeasure. PLoS ONE, 10(12), e0143263. https://doi.org/10.1371/journal.pone.0143263

Koriat, A. (2008). When confidence in a choice is independent of which choice is made. Psychonomic Bulletin \& Review, 15, 997-1001. https://doi.org/10.3758/PBR.15.5.997 Koriat, A., \& Goldsmith, M. (1996). Monitoring and control processes in the strategic regulation of memory accuracy. Psychological Review, 103(3), 490-517. https://doi.org/10.1037/0033-295X.103.3.490

Kozyreva, A., \& Hertwig, R. (2021). The interpretation of uncertainty in ecological rationality. Synthese, 198(2), 1517-1547. https://doi.org/10.1007/s11229-019-02140-w

Kuvaas, B., \& Kaufmann, G. (2004). Impact of mood, framing, and need for cognition on decision makers' recall and confidence. Journal of Behavioral Decision Making, 17(1), 59-74. https://doi.org/10.1002/bdm.461

Landrø, M., Pfuhl, G., Engeset, R., Jackson, M., \& Hetland, A. (2020). Avalanche decisionmaking frameworks: Classification and description of underlying factors. Cold Regions Science and Technology, 169, 102903. https://doi.org/10.1016/j.coldregions.2019.102903

Lerner, J. S., Li, Y., Valdesolo, P., \& Kassam, K. S. (2015). Emotion and decision making. Annual Review of Psychology, 66(1), 799-823. https://doi.org/10.1146/annurev-psych010213-115043

Loewenstein, G. F., Weber, E. U., Hsee, C. K., \& Welch, N. (2001). Risk as feelings. Psychological Bulletin, 127(2), 267-286. https://doi.org/10.1037/0033-2909.127.2.267 
Løhre, E., \& Teigen, K. H. (2016). There is a $60 \%$ probability, but I am $70 \%$ certain: communicative consequences of external and internal expressions of uncertainty. Thinking \& Reasoning, 22(4), 369-396. https://doi.org/10.1080/13546783.2015.1069758

Massoni, S. (2014). Emotion as a boost to metacognition: How worry enhances the quality of confidence. Consciousness and Cognition, 29, 189-198. https://doi.org/10.1016/j.concog.2014.08.006

Meyniel, F., Schlunegger, D., \& Dehaene, S. (2015). The sense of confidence during probabilistic learning: a normative account. PLOS Computational Biology, 11(6), e1004305. https://doi.org/10.1371/journal.pcbi.1004305

Nalborczyk, L., Batailler, C., Loevenbruck, H., Vilain, A., \& Bürkner, P. (2019, May 14). An introduction to Bayesian multilevel models using brms: a case study of gender effects on vowel variability in standard Indonesian. PsyArXiv. https://doi.org/10.1044/2018_JSLHR-S-18-0006

Peterson, D. K., \& Pitz, G. F. (1988). Confidence, uncertainty, and the use of information. Journal of Experimental Psychology: Learning, Memory, and Cognition, 14(1), 85-92. https://doi.org/10.1037/0278-7393.14.1.85

Petrusic, W.M., \& Baranski, J.V. (2003). Judging confidence influences decision processing in comparative judgments. Psychonomic Bulletin \& Review, 10, 177-183. https://doi.org/10.3758/BF03196482

Prinz, A., Bergmann, V., \& Wittwer, J. (2019). Happy but overconfident: positive affect leads to inaccurate metacomprehension. Cognition and Emotion, 33(3), 606-615. https://doi.org/10.1080/02699931.2018.1472553

R Core Team (2017). $R$ : A language and environment for statistical computing. R Foundation for Statistical Computing, Vienna, Austria. 
Sanchez, C., \& Dunning, D. (2018). Overconfidence among beginners: Is a little learning a dangerous thing? Journal of Personality and Social Psychology, 114(1), 10-28. https://doi.org/10.1037/pspa0000102

Sanchez, C., \& Dunning, D. (2020). Decision fluency and overconfidence among beginners. Decision, 7(3), 225-237. https://doi.org/10.1037/dec0000122

Schwarz, N. (2012). Feelings-as-information theory. In P. A. M. Van Lange, A. W. Kruglanski, \& T. Higgins (Eds.), Handbook of Theories of Social Psychology: Volume 1 (pp. 289-308). London: Sage Publications Ltd.

Sidi, Y., Ackerman, R., \& Erez, A. (2018). Feeling happy and (over)confident: the role of positive affect in metacognitive processes. Cognition and Emotion, 32(4), 876-884. https://doi.org/10.1080/02699931.2017.1346501

Simonsohn, U. (2018). Two Lines: A valid alternative to the invalid testing of u-shaped relationships with quadratic regressions. Advances in Methods and Practices in Psychological Science, 1(4), 538-555. https://doi.org/10.1177/2515245918805755

Slovic, P. (1987). Perception of risk. Science, 236(4799), 280. https://doi.org/10.1126/science.3563507

Slovic, P., Finucane, M., Peters, E., \& MacGregor, D. G. (2002). The affect heuristic. In D. Griffin, D. Kahneman, \& T. Gilovich (Eds.), Heuristics and Biases: The Psychology of Intuitive Judgment (pp. 397-420). Cambridge: Cambridge University Press.

Slovic, P., Finucane, M. L., Peters, E., \& MacGregor, D. G. (2004). Risk as analysis and risk as feelings: some thoughts about affect, reason, risk, and rationality. Risk Analysis, 24(2), 311-322. https://doi.org/10.1111/j.0272-4332.2004.00433.x

Stephensen, M. B., \& Martiny-Huenger, T. (2021). Liking and perceived safety across judgments of distinct instances of a category of activity. Journal of Risk Research, 1-17. https://doi.org/10.1080/13669877.2021.1905693 
Teigen, K. H., \& Løhre, E. (2017). Expressing (un)certainty in no uncertain terms: reply to Fox and Ülkümen. Thinking \& Reasoning, 23(4), 492-496. https://doi.org/10.1080/13546783.2017.1314965

Ülkümen, G., Fox, C. R., \& Malle, B. F. (2016). Two dimensions of subjective uncertainty: Clues from natural language. Journal of Experimental Psychology: General, 145(10), 1280-1297. https://doi.org10.1037/xge0000202

Utah Avalanche Center. (n.d.). Forecasts. Retrieved from https://utahavalanchecenter.org/ Varsom. (n.d.). Avalanche Bulletins for Norway. Retrieved from http://www.varsom.no/en/avalanche-bulletins/

Yeung, N., \& Summerfield, C. (2012). Metacognition in human decision-making: confidence and error monitoring. Philosophical Transactions of the Royal Society B: Biological Sciences, 367(1594), 1310-1321. https://doi.org10.1098/rstb.2011.0416

Zweifel, B., \& Haegeli, P. (2014). A qualitative analysis of group formation, leadership and decision making in recreation groups traveling in avalanche terrain. Journal of Outdoor Recreation and Tourism, 5-6, 17-26. https://doi.org/10.1016/j.jort.2014.03.001 


\section{Supplemental Materials}

\section{Table 1}

Results of linear regression analysis of the relation between experience and judged safety per scenario used in Studies 1 to 4, and the results Breusch-Pagan tests of non-constant variance for that linear relation. The null hypothesis for the Breusch-Pagan test assumes homoscedasticity of variance.

\begin{tabular}{rcccccc}
\hline \multirow{2}{*}{ Scenario } & \multicolumn{3}{c}{ Judged safety $~$} & & \multicolumn{2}{c}{ Breusch-Pagan test $(\mathrm{df}=1)$} \\
\cline { 2 - 3 } & $b_{\text {experience }}(S E)$ & $95 \% C I$ & $p$-value & & $\chi^{2}$ & $p$-value \\
\hline 1 & $-.15(.06)$ & $-.26,-.04$ & .007 & & 3.69 & .055 \\
2 & $.01(.05)$ & $-.10, .11$ & .864 & & 1.02 & .313 \\
3 & $-.08(.07)$ & $-.22, .06$ & .249 & & .17 & .684 \\
4 & $-.01(.05)$ & $-.11, .09$ & .858 & & .64 & .424 \\
5 & $-.02(.06)$ & $-.15, .11$ & .761 & & .01 & .927 \\
6 & $.00(.06)$ & $-12, .11$ & .950 & & .00 & .965 \\
7 & $-.03(.14)$ & $-.30, .25$ & .835 & & .37 & .544 \\
8 & $-.06(.12)$ & $-.31, .18$ & .603 & & .63 & .426 \\
9 & $-.32(.22)$ & $-78, .13$ & .155 & & .89 & .346 \\
10 & $-.12(.18)$ & $-.51, .26$ & .505 & & .05 & .817 \\
11 & $.06(.20)$ & $-.34, .47$ & .746 & & .38 & .535 \\
12 & $.02(.14)$ & $-.26, .31$ & .859 & & .16 & .688 \\
\hline
\end{tabular}

